\title{
Auf die Prozesse kommt es an
}

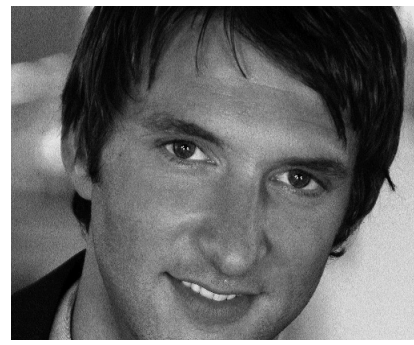

VON MARKUS SCHMID Markus Schmid ist Mitarbeiter der PRX Agentur für Public Relations in Stuttgart. Der Beitrag ist die Zusammenfassung eines Vortrags von Prof. Helmut Kreidenweis von der Katholischen Universität Eichstätt-Ingolstadt auf dem Infotag 2010 der Diamant Software $\mathrm{GmbH} \&$ Co. KG für das Gesundheits- und Sozialwesen. Internet http://www.pr-x.de

\author{
Zur Steigerung der Wertschöpfung kann in sozialen \\ Organisationen die Informationstechnik erheblich \\ beitragen. Noch werden allerdings Computer und Co. zu \\ wenig in die Gestaltung von Geschäftsprozessen \\ einbezogen, wie das nachfolgende Resümee eines \\ Vortrags von Prof. Helmut Kreidenweis zeigt.
}

Wollen soziale Einrichtungen die Wertschöpfung ihrer Informationstechnik (IT) steigern, müssen sie zunächst einmal identifizieren, wo diese überhaupt stattfindet.

Aus betriebswirtschaftlicher Sicht lassen sich drei wesentliche Typen von Geschäftsprozessen unterscheiden: Das sind erstens die Leistungsprozesse, wie beispielsweise Aufnahme, Betreuung und Entlassung. Daneben existieren zweitens Unterstützungsprozesse wie Leistungsdokumentation und Leistungsabrechnung, fachliche Dokumentation oder Finanzbuchhaltung. Den dritten Typus bilden die Managementprozesse wie strategische Planung, Personalmanagement oder Finanzierung. Die eigentliche Wertschöpfung findet dabei in den Leistungsprozessen statt. Ausschließlich hier erbringen soziale Einrichtungen ihre originären Leistungen (vgl. Abb. »Wo findet die Wertschöpfung statt? «).

Aber auch wenn die Unterstützungsprozesse keinen direkten Wertschöpfungsbeitrag leisten, liegt hier der Hauptfokus der Informationstechnik. Denn: Potenziale, die an die oftmals personalintensiven Unterstützungsprozesse gebunden sind, können von der Informationstechnik für die Leistungsprozesse freigelegt werden - und damit für diejenigen Abläufe, in denen die eigentliche Wertschöpfung von Sozialunternehmen stattfindet. Wenn etwa die Stationsoder Pflegedienstleitung viele Stunden damit zubringt, für das Personalbüro die Zeitzuschläge ihrer Mitarbeiter zu berechnen, dann ist das Arbeitszeit, die von ihren Führungsaufgaben abgeht. Das ist keinesfalls Wertschöpfung, sondern Wertvernichtung.

\section{Warum die Anwendungssoftware im Blickpunkt stehen sollte}

Wo genau also kann die Informationstechnik wertschöpfend wirken? Betrachtet man die Informationstechnologie in sozialen Einrichtungen oder Krankenhäusern, findet man dabei vier wesentliche Säulen:

- Die IT-Infrastruktur sorgt dafür, dass die Server laufen, die Netzwerke funktionieren und die Systeme performant arbeiten.

- Eine wichtige Rolle spielt die IT-Sicherheit, angesichts der sehr sensiblen Daten im Sozialwesen.

- Die IT-Organisation beschäftigt sich damit, wo die Informationstechnik organisatorisch verankert ist, wie viele Mitarbeitende benötigt werden usw.

- Die Anwendungssoftware schließlich bildet die vierte Säule der Informationstechnik in Sozialunternehmen.

Die ersten drei Bereiche sind primär das Geschäft des IT-Leiters. Der Sozialmanager dagegen sollte seinen Blick eindeutig auf die Anwendungssoftware 


\section{Wo findet die Wertschöpfung statt?}

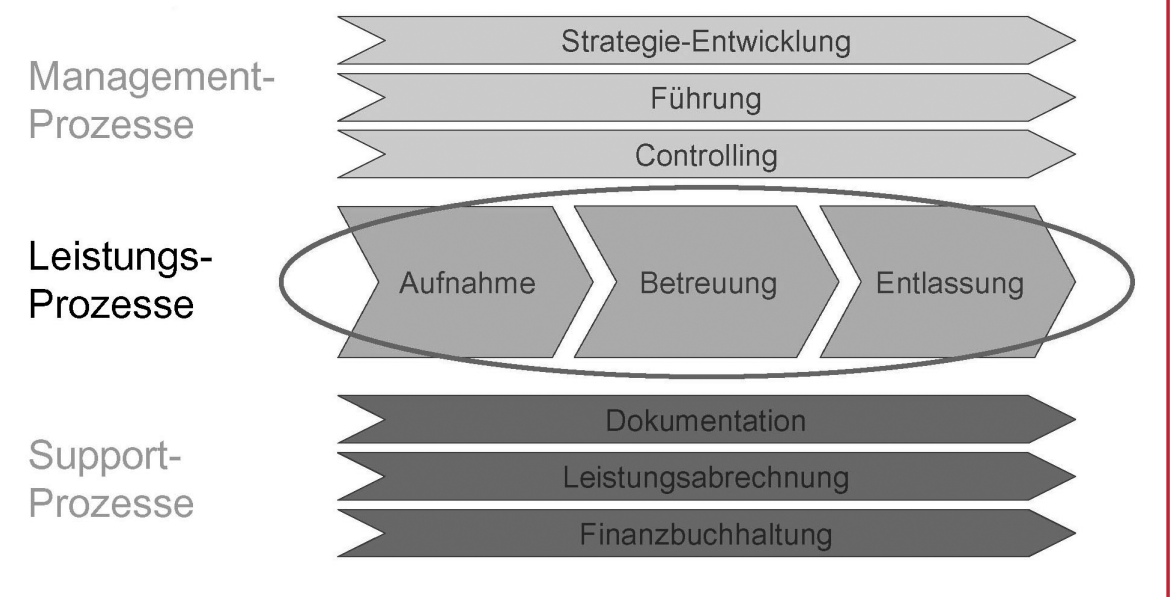

richten. Denn das ist diejenige Säule, die innerhalb des gesamten IT-Komplexes am meisten zur Wertschöpfung beiträgt. Hier werden die Prozesse abgebildet, die bei den Mitarbeitenden entweder als Hindernis oder als Unterstützung wirken. Die ersten drei Säulen ganz bewusst als Technologie zu sehen, bedeutet nicht zuletzt auch eine Entlastung für das Management. Denn diese Aufgaben kann das Management der IT-Leitung überlassen. Was aber auf keinen Fall delegiert werden sollte, ist die vierte Säule. Sie ist aus den genannten Gründen eindeutig als Managementaufgabe zu betrachten.
Innerhalb der Anwendungssoftware liegt der strategische Wertschöpfungsfokus bei den fachspezifischen Systemen. Der Grund: Hier findet das Massengeschäft statt, hier werden die größten Personalressourcen eingesetzt, die dort sitzen, wo die eigentlichen Leistungen der Sozialunternehmen erbracht werden. Entsprechend groß ist momentan der Zuwachs in den Einrichtungen bei den Fachsystemen für Dienst-, Einsatz- und Hilfeplanung sowie Dokumentation. Die zentralen Bereiche sind längst mit Informationstechnik gesättigt und weisen bereits eine Eins-zu-eins-Durchdringung mit Informationstechnik auf,

\section{Wertschöpfung auf Ebene des Anwendungssoftware-Portfolios}

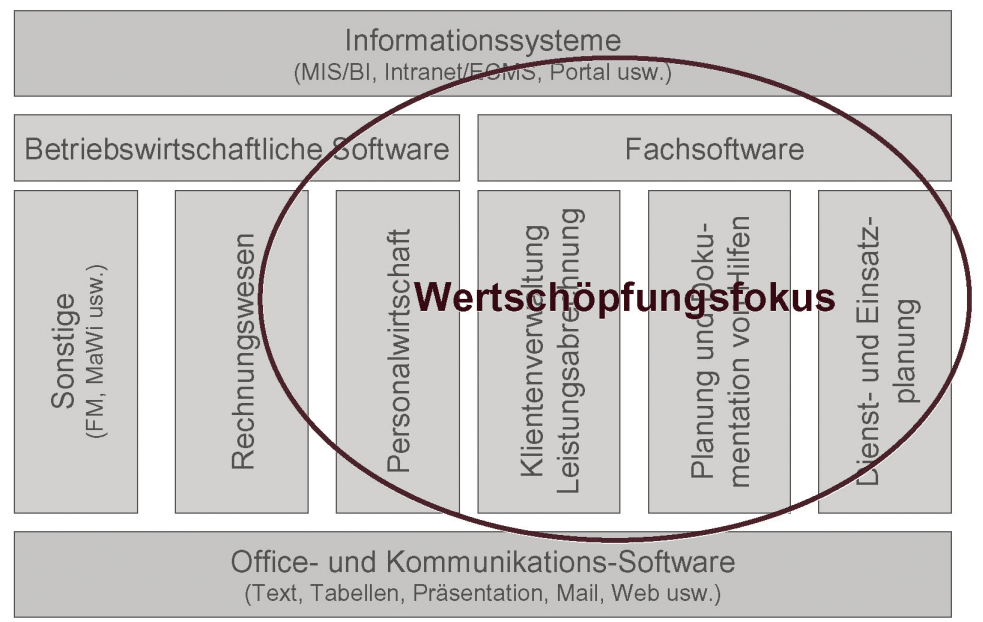

sprich: Jeder Mitarbeiter in der zentralen Verwaltung verfügt über einen eigenen PC. Jetzt wird hinsichtlich der Fachsysteme nachgezogen (vgl. Abb. »Wertschöpfung auf Ebene des Anwendungssoftware-Portfolios «).

Der strategische Wertschöpfungsfokus reicht jedoch über die Fachsysteme hinaus und berührt auch Gebiete wie Personalwirtschaft und Rechnungswesen. Bei Ersterem gilt das vor allem für die arbeits- und zeitintensiven Aufgaben wie Personalmanagement, Bewerberverwaltung, Einstellungsverfahren oder Auskunftserteilung. Auch hier findet sich ein Wertschöpfungsbeitrag der Informationstechnik, der sich steigern lässt. Dasselbe gilt für Massen- und Routinearbeiten im Rechnungswesen wie Kassenverwaltung, Mahnwesen oder beispielsweise Operationsverwaltung im Krankenhaus.

\section{Wie die Informationstechnik zur Wertschöpfung beitragen kann}

Wie beurteilen die Sozialunternehmen selbst den Wertbeitrag der Informationstechnik? Mit diesem Thema beschäftigt sich schwerpunktmäßig der letztjährige »IT-Report für die Sozialwirtschaft «, eine regelmäßig von der Arbeitsstelle für Sozialinformatik der Katholischen Universität Eichstätt-Ingolstadt durchgeführte empirische Untersuchung. In der Ausgabe 2010 wurden die Einrichtungen unter anderem gefragt, welche Ziele sie mit dem Einsatz von Informationstechnik verfolgen. Überraschendes Ergebnis: Nicht Kosteneinsparungen oder Erlössteigerungen sind heute das oberste Ziel der Verantwortlichen, sondern vielmehr die Steigerung der fachlichen Qualität! Arbeitsabläufe effizienter zu gestalten - und damit der Kostenaspekt - folgt erst mit knappem Abstand auf Platz zwei. Wiederum knapp dahinter ist das drittwichtigste Ziel, die Entscheidungsqualität durch Verfügbarkeit von Daten zu verbessern.

Weniger überraschend ist dagegen, wie die Potenziale der Informationstechnik in verschiedenen zentralen Geschäftsprozessen ausgeschöpft werden. Die besten Werte finden sich hier in den klassischen verwaltungsgetriebenen Prozessen. Absoluter Spitzenreiter ist das Rechnungswesen. Schon etwas schlechter sieht es hinsichtlich der Aus- 
schöpfung der IT-Potenziale im Bereich Klientenverwaltung und Leistungsabrechnung aus. Beinahe schon dramatisch ist die Lage auf den Gebieten Dienst-, Einsatz- und Hilfeplanung sowie Dokumentation. Hier gibt lediglich die Hälfte der Befragten an, die Potenziale gut auszuschöpfen.

Als Fazit lässt sich festhalten: In den klassischen Verwaltungsprozessen sieht es deutlich besser aus als in den Leistungsprozessen. Interessant: Bei der Potenzialausschöpfung sind kaum Unterschiede feststellbar zwischen den verschiedenen Arbeitsfeldern wie Behinderten-, Alten-, Suchtkranken- oder Kinder- und Jugendhilfe. Eine deutliche Botschaft gibt es aber hinsichtlich der Unternehmensgröße: Die Potenzialausschöpfung ist bei größeren Organisationen deutlich höher. Geradezu einen Einbruch gibt es dabei an der Grenze zu Trägern mit weniger als 100 Mitarbeitern. Hier fallen die Zahlen extrem ab (vgl. Abb. »Inwieweit werden derzeit Ihrer Meinung nach in Ihrer Organisation die Potenziale der IT ausgeschöpft? «).

Ein weiterer in der Studie untersuchter Faktor ist die Kostentransparenz in der Informationstechnik. Ergebnis: Gut die Hälfte kennt die IT-Kosten vollständig, die andere nur teilweise oder gar nicht. Auch hier steigt der Wert mit zunehmender Unternehmensgröße deutlich, was natürlich auf den entsprechenden Reifegrad von Rechungswesen und Controlling zurückzuführen ist. Recht positiv beurteilt wird von den Befragten insgesamt das Kosten-Nutzen-Verhältnis der Informationstechnik.

Aufschlussreich sind die Antworten auf die Frage, wie die Informationstechnik in der Organisation aufgestellt ist. Lediglich der Betreiber technischer Systeme? Eher ein dienstleistungsorientierter Partner? Oder kümmert sie sich sogar aktiv um die Gestaltung der Geschäftsprozesse? Das deutliche Ergebnis: Die Informationstechnik wird primär als Betreiber technischer Systeme wahrgenommen, nur teilweise als Partner, und noch einmal deutlich schwächer als Gestalter der Geschäftsprozesse.

Unter dem Strich lassen sich aus den erhobenen Daten einige Faktoren ableiten, welche den Wertschöpfungsbeitrag der Informationstechnik positiv beeinflussen. Besonders auffällig dabei: Einrichtungen, die über eine fixierte IT-

\section{Inwieweit werden derzeit lhrer Meinung nach in Ihrer Organisation die Potenziale der IT ausgeschöpft?}

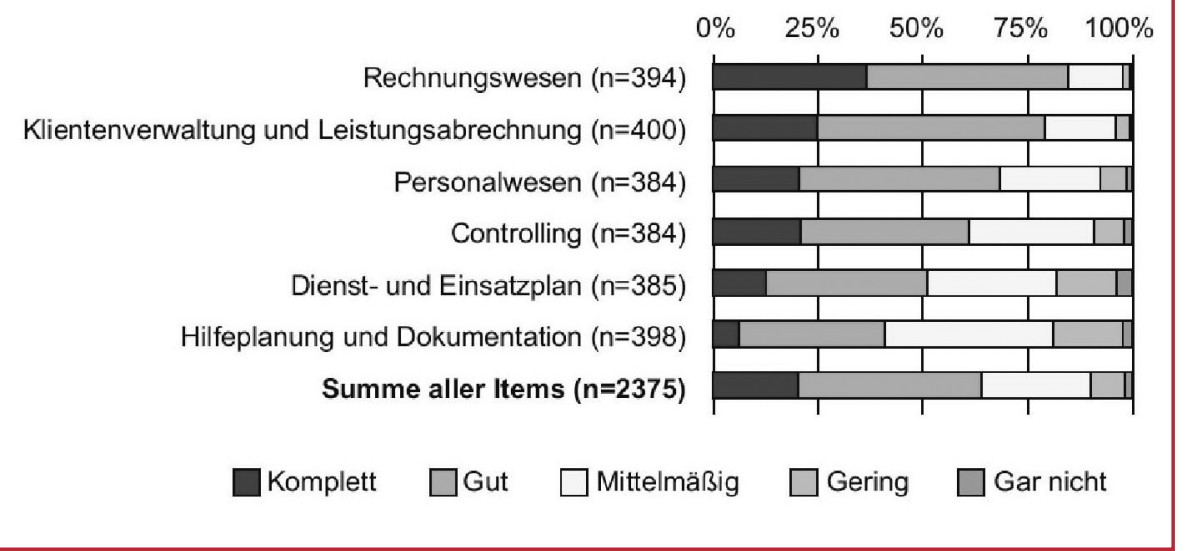

Strategie verfügen, erreichen ihre Ziele eher und können ihre Potenziale besser ausschöpfen. Auch Organisationen, die eine hohe Kostentransparenz aufweisen, sind beim Erreichen ihrer Ziele erfolgreicher. Dieselbe Korrelation zeigt sich bei Einrichtungen, die in der Informationstechnik einen Partner mit Prozesskompetenz sehen. Last, but not least treten hierbei auch Skaleneffekte auf: Große Träger sind hinsichtlich Potenzialausschöpfung und Zielerreichung besser.

\section{Was Sozialmanager tun können}

Sowohl aus den Daten als auch der praktischen Erfahrung zeigt sich: Geht es um die Entwicklung einer wertschöpfenden IT-Strategie in Sozialunterneh- men, müssen die Einrichtungen zuallererst formulieren, was von der Informationstechnik erwartet wird.

Gefragt ist hierbei nicht nur die oberste, sondern vor allem auch die zweite Führungsebene: Was konkret muss die Informationstechnik leisten? Diese Frage ist mit dem primären Fokus auf die Anwendungssysteme zu beantworten. Entscheidend dabei: die Business-Perspektive der Informationstechnik! Daraus leitet sich das Anwendungsportfolio $\mathrm{ab}$, das natürlich möglichst medienbruchfrei und prozessorientiert gestaltet sein muss. Daraus wiederum ergibt sich die IT-Organisation und erst dann kommt die Ebene der Infrastruktur und IT-Sicherheit (vgl. Abb. »Wege zu einer wertschöpfenden IT-Strategie-Entwicklung «).

\section{Wege zu einer wertschöpfenden IT-Strategie-Entwicklung}

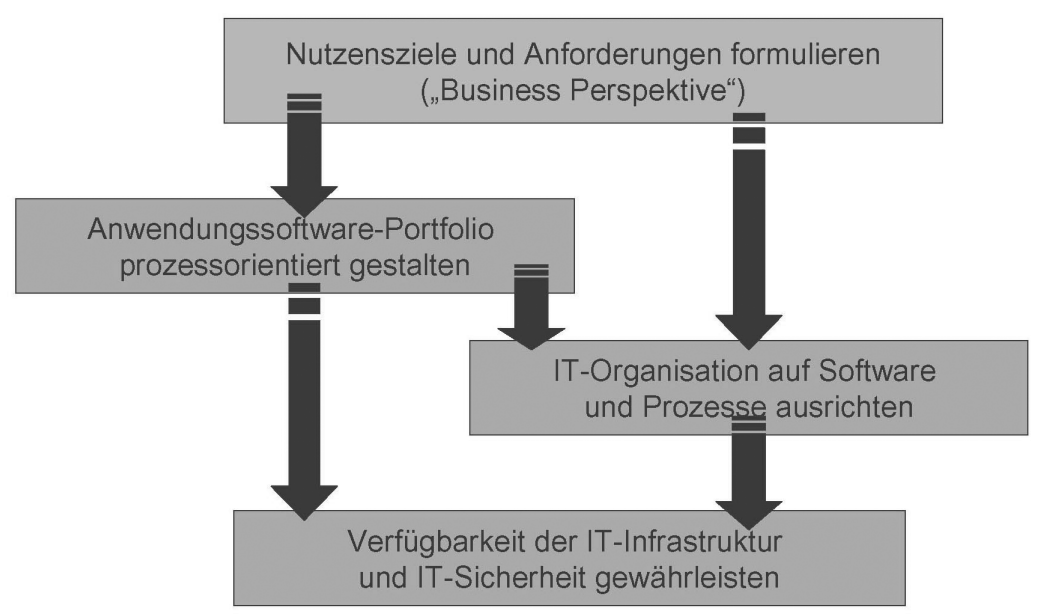


Generell lassen sich als Handlungsempfehlungen für Sozialmanager folgende Punkte hervorheben:

- Informationstechnik als Managementthema begreifen: Natürlich ist Informationstechnik nicht das wichtigste Managementthema. Dennoch: Die oberste, aber gerade auch die zweite Führungsebene kann sich vor diesem Thema nicht drücken.

- Informationstechnik, Qualitätsmanagement und Organisationsgestaltung vernetzen: Diese drei Bereiche sind in sozialen Einrichtungen häufig
Hilfreich kann es etwa sein, regelmäßig die Durchdringungsquoten der Fachbereiche zu ermitteln, die Kostenquoten oder auch die Dienstleistungsqualität der Informationstechnik.

- Informationstechnik in die Entwicklung der Unternehmensstrategie einbinden: Wird die Informationstechnik von Beginn an in strategische Überlegungen eingebunden, kann sie beratend zur Seite stehen und unter Umständen sogar ganz neue Perspektiven eröffnen. Etwa bei dem Vorhaben, Verantwortung zu dezentralisieren und in die Leistungsbereiche zu

\section{"Nicht Kosteneinsparungen oder Erlös- steigerungen sind oberste Ziele bei der Informationstechnik, sondern die Steigerung der fachlichen Qualität"}

Parallelwelten. Es läuft die Auswahl einer Dienstplanungssoftware, und der Qualitätsmanager hat noch nie etwas davon gehört. Aber: Die Frage: »Wie können wir kunden- und mitarbeiterorientierte Dienstpläne gestalten? " hat sehr viel mit Qualität zu tun! Was spricht deshalb dagegen, diese drei Bereiche organisatorisch zusammenzulegen - beispielsweise in einem Kompetenzzentrum »Organisation, Qualitätsmanagement und Informationstechnik «? Eine Konstellation, die man in der freien Wirtschaft bereits häufiger vorfindet.

- Transparenz durch IT-Reporting schaffen: Hier ist einerseits die Informationstechnik selbst gefragt, die nötige Transparenz herzustellen, aber auch das Management, diese einzufordern. verlagern. Ein solches Projekt hat viel mit Informationstechnik zu tun: Wie wollen die Leute vor Ort mit Informationen versorgt werden? Welche Informationen müssen sie auf welchem Wege an die Zentrale schicken? Die Informationstechnik kann dann vielleicht bereits an dieser Stelle auf ganz neue Chancen der Informationsversorgung hinweisen, die es ermöglichen, noch weit mehr Bereiche in die dezentrale Verantwortung zu geben, als ursprünglich geplant war.

- IT-Strategie-Entwicklung einleiten: Im Grunde geht es bei der IT-Strategie weniger um das Ergebnis in Form eines offiziellen Papiers. Wesentlich wichtiger ist der Kommunikationsprozess, in dem das Papier entsteht, die Verständigung darüber, was erreicht werden soll. Wichtig dabei: auch die zweite und dritte Ebene einbinden.

- IT-Projektmanagement etablieren: Immer wenn es darum geht, neue Lösungen auszuwählen oder einen Bereich neu auszustatten, ist es ratsam, ein vernünftiges Projektmanagement aufzusetzen. In der Praxis erlebt man dagegen häufig ein wachsweiches Projektmanagement: Es gibt zwar einen Projektantrag und auch ein Projektplan wird erstellt. Ein wirkliches Projekt-Controlling ist aber nicht vorhanden, eine ordentliche Berichterstattung fehlt ebenso wie klare Entscheidungsstrukturen.

- Kleinere und mittlere Träger sollten Skaleneffekte durch IT-Verbünde nutzen: Die deutlichen Skaleneffekte bei der Informationstechnik bedeuten, dass kleinere und mittlere Träger an Wettbewerbsfähigkeit verlieren. Auch wenn dies nicht im ganz großen Maßstab der Fall ist, ein Beitrag ist es allemal, und dieser wird angesichts der zunehmenden Durchdringung mit Informationstechnik in Zukunft an Bedeutung gewinnen. Was können kleinere und mittlere Träger also tun, um sich aufzustellen wie die Großen? Die Antwort kann nur lauten: sich entweder zusammentun oder outsourcen. Letzteres ist natürlich immer problematisch, weil Kompetenzen nach außen gegeben werden. Im Heimbereich kann das aber sinnvoll sein, etwa wenn es um den bloßen Betrieb der Hardware geht. Bei ersterem liegt ein Teil der Verantwortung bei den Verbänden. Bezirks- oder Kreisverbände müssen kleine Träger besser unterstützen, wenn die breite und bunte Landschaft sozialer Träger weiterhin aufrecht erhalten bleiben soll. Bei den Einrichtungen selbst ist dieses Thema bereits angekommen, die Verbandsebene hat es bisher noch nicht erreicht.

\section{Die Arbeitsstelle für Sozialinformatik}

an der Katholischen Universität Eichstätt-Ingolstadt will den Einsatz von Informationstechnik in den Feldern der Sozialen Arbeit und im Management sozialer Organisationen fördern und diese Prozesse kritisch begleiten. Zu den Aufgaben der Arbeitsstelle gehören die Durchführung von Forschungs-, Entwicklungs- und Evaluationsprojekten sowie die Praxisberatung.

Internet http://www.sozialinformatik.de

\section{Die Diamant Software}

ist seit mehr als 30 Jahren der Spezialist für Rechnungswesenund Controlling-Software. Über 4.000 Kunden - davon mehr als 150 Krankenhäuser und Kliniken sowie über 450 soziale Einrichtungen und Wohlfahrtsverbände-arbeiten mit den Lösungen des Unternehmens. Im Vordergrund steht dabei die Integration in die Systemlandschaft und in die Prozesse der Organisationen.

Internet http://www.diamant-software.de 